\title{
A proof of the peak polynomial positivity conjecture
}

\author{
Alexander Diaz-Lopez ${ }^{* 1}$, Pamela E. Harris ${ }^{\dagger 2}$, Erik Insko ${ }^{\ddagger 3}$ and Mohamed Omar ${ }^{\S 4}$ \\ ${ }^{1}$ Department of Mathematics and Statistics, Swarthmore College \\ ${ }^{2}$ Department of Mathematics and Statistics, Williams College \\ ${ }^{3}$ Department of Mathematics, Florida Gulf Coast University \\ ${ }^{4}$ Department of Mathematics, Harvey Mudd College
}

\begin{abstract}
We say that a permutation $\pi=\pi_{1} \pi_{2} \cdots \pi_{n} \in \mathfrak{S}_{n}$ has a peak at index $i$ if $\pi_{i-1}<\pi_{i}>\pi_{i+1}$. Let $P(\pi)$ denote the set of indices where $\pi$ has a peak. Given a set $S$ of positive integers, we define $P(S ; n)=\left\{\pi \in \mathfrak{S}_{n}: P(\pi)=S\right\}$. In 2013 Billey, Burdzy, and Sagan showed that for subsets of positive integers $S$ and sufficiently large $n,|P(S ; n)|=p_{S}(n) 2^{n-|S|-1}$ where $p_{S}(x)$ is a polynomial depending on $S$. They gave a recursive formula for $p_{S}(x)$ involving an alternating sum, and they conjectured that the coefficients of $p_{S}(x)$ expanded in a binomial coefficient basis centered at $\max (S)$ are all nonnegative. In this paper we introduce a new recursive formula for $|P(S ; n)|$ without alternating sums and we use this recursion to prove that their conjecture is true.
\end{abstract}

Keywords: binomial coefficient, peaks, peak polynomial, permutation, positivity conjecture.

\section{Introduction}

Let $[n]:=\{1,2, \ldots, n\}$ and let $\mathfrak{S}_{n}$ denote the symmetric group on $n$ letters. Let $\pi=\pi_{1} \pi_{2} \cdots \pi_{n}$ denote the one-line notation for $\pi \in \mathfrak{S}_{n}$. We say that $\pi$ has a peak at index $i$ if $\pi_{i-1}<\pi_{i}>\pi_{i+1}$ and define the peak set of a permutation $\pi$ to be the set:

$$
P(\pi)=\{i \in[n] \mid \pi \text { has a peak at } i\} .
$$

Given a subset $S \subseteq[n]$ we denote the set of all permutations with peak set $S$ by

$$
P(S ; n)=\left\{\pi \in \mathfrak{S}_{n} \mid P(\pi)=S\right\} .
$$

Whenever $P(S ; n) \neq \emptyset$, we say $S \subseteq[n]$ is $n$-admissible or simply admissible when the $n$ is understood. If $S$ is $n$-admissible, then it is $k$-admissible for any $k \geq n$.

Billey, Burdzy, and Sagan first studied the subsets $P(S ; n) \subseteq \mathfrak{S}_{n}$ for $n$-admissible sets $S$ in 2013 [3]. Their work was motivated by a problem in probability theory which explored the relationship

\footnotetext{
*adiazlo1@swarthmore.edu

pamela.e.harris@williams.edu

einsko@fgcu.edu

omar@g.hmc.edu
}

(C) 2017. This manuscript version is made available under the Elsevier user license 
between mass distribution on graphs and random permutations with specific peak sets [2]. One of their foundational results established that for an $n$-admissible set $S$

$$
|P(S ; n)|=p_{S}(n) 2^{n-|S|-1}
$$

where $p_{S}(x)$ is a polynomial depending on $S$, which they called the peak polynomial of $S$. It was shown that $p_{S}(x)$ has degree $\max (S)-1$ when $S \neq \emptyset, p_{S}(x)=1$ when $S=\emptyset, p_{S}(x)=0$ when $S$ is non-admissible, and that $p_{S}(x)$ takes on integral values when evaluated at integers [3, Theorem 1]. Similar observations were made for peak polynomials in other classical Coxeter groups (see the work of Castro-Velez, Diaz-Lopez, Orellana, Pastrana, and Zevallos [8] and Diaz-Lopez, Harris, Insko, and Perez-Lavin [9]). Using the method of finite differences, Billey, Burdzy, and Sagan gave closed formulas for the peak polynomials $p_{S}(x)$ in various special cases. The finite forward difference operator $\Delta$ is a linear operator defined by $(\Delta f)(x)=f(x+1)-f(x)$. Iterating this operator gives higher order differences defined by

$$
\left(\Delta^{j} f\right)(x)=\left(\Delta^{j-1} f\right)(x+1)-\left(\Delta^{j-1} f\right)(x),
$$

for $j \geq 1$, where $\left(\Delta^{0} f\right)(x)=f(x)$. Using Newton's forward difference formula, Billey, Burdzy, and Sagan expanded $p_{S}(x)$ in the binomial basis centered at $m=\max (S)$ as

$$
p_{S}(x)=\sum_{j=0}^{m}\left(\Delta^{j} p_{S}\right)(m)\left(\begin{array}{c}
x-m \\
j
\end{array}\right)
$$

and conjectured that for any admissible set $S$ with $m=\max (S)$ each coefficient $\left(\Delta^{j} p_{S}\right)(m)$ is a positive integer for $1 \leq j \leq m-1$ [3, Conjecture 14]. This conjecture has become known as the positivity conjecture for peak polynomials.

Example 1.1. Below is a table of forward differences for the peak polynomial $p_{\{4,6\}}(x)$. The $(j, k)$ entry in this table is the coefficient $\left(\Delta^{j} p_{S}\right)(k)$ of $\left(\begin{array}{c}x-k \\ j\end{array}\right)$ in the expansion of $p_{S}(x)$ in the binomial basis centered at $k$.

\begin{tabular}{|l|rrrrrrr|}
\hline$j, k$ & 0 & 1 & 2 & 3 & 4 & 5 & 6 \\
\hline 0 & 4 & 2 & 2 & 2 & 0 & -3 & 0 \\
1 & -2 & 0 & 0 & -2 & -3 & 3 & 25 \\
2 & 2 & 0 & -2 & -1 & 6 & 22 & 50 \\
3 & -2 & -2 & 1 & 7 & 16 & 28 & 43 \\
4 & 0 & 3 & 6 & 9 & 12 & 15 & 18 \\
5 & 3 & 3 & 3 & 3 & 3 & 3 & 3 \\
6 & 0 & 0 & 0 & 0 & 0 & 0 & 0 \\
\hline
\end{tabular}

Table 1: Forward difference table for the peak polynomial $p_{\{4,6\}}(x)$.

For example, we expand $p_{\{4,6\}}(x)$ in the binomial bases centered at 0 and 6 as

$$
\begin{aligned}
p_{\{4,6\}}(x) & =4\left(\begin{array}{l}
x \\
0
\end{array}\right)-2\left(\begin{array}{l}
x \\
1
\end{array}\right)+2\left(\begin{array}{l}
x \\
2
\end{array}\right)-2\left(\begin{array}{l}
x \\
3
\end{array}\right)+0\left(\begin{array}{l}
x \\
4
\end{array}\right)+3\left(\begin{array}{l}
x \\
5
\end{array}\right)+0\left(\begin{array}{l}
x \\
6
\end{array}\right) \\
& =0\left(\begin{array}{c}
x-6 \\
0
\end{array}\right)+25\left(\begin{array}{c}
x-6 \\
1
\end{array}\right)+50\left(\begin{array}{c}
x-6 \\
2
\end{array}\right)+43\left(\begin{array}{c}
x-6 \\
3
\end{array}\right)+18\left(\begin{array}{c}
x-6 \\
4
\end{array}\right)+3\left(\begin{array}{c}
x-6 \\
5
\end{array}\right)+0\left(\begin{array}{c}
x-6 \\
6
\end{array}\right) .
\end{aligned}
$$


Billey, Burdzy, and Sagan proved the positivity conjecture holds when $|S| \leq 1$ [3, Proposition 16], verified it computationally for all $2^{m}$ subsets containing a largest value $m=\max (S)=20$, and showed that $p_{S}(m)=0$ for any set $S$ [3, Lemma 15]. In 2014, Billey, Fahrbach, and Talmage posed a stronger conjecture bounding the moduli of the roots of $p_{S}(z)$, which they checked numerically for all peak sets with $\max (S) \leq 15$ [4, Conjecture 1.6]. They also discovered a computationally efficient recursive algorithm for computing $p_{S}(x)$, and showed that $p_{S}(k)>0$ for $k>m$ and that the positivity conjecture holds in several special cases, including when the position of the last peak of $S$ is three more than the position of the penultimate peak [4, Lemmas 4.4 and 3.9].

Our main result is the following theorem, which proves the positivity conjecture in all cases.

Theorem 1.2. If $S \subseteq[n]$ is a nonempty admissible set with $m=\max (S)$, then $\left(\Delta^{j} p_{S}\right)(k)>0$ for all $1 \leq j \leq m-1$ and $k \geq m$, and $\left(\Delta^{m} p_{S}\right)(x)=0$.

We prove Theorem 1.2 at the end of Section 2. Positivity of coefficients in a given binomial basis is a phenomenon that occurs throughout combinatorics. A particular illuminating example comes from Ehrhart theory. For a $d$-dimensional integral convex polytope $P$, recall that $i_{P}(n)$ is the number of integer points in the $n$-th dilation of $P$. Ehrhart proved that $i_{P}(n)$ is a polynomial in $n$ of degree $d$, so classical techniques in generating functions establish that $i_{P}(n)=\sum_{j=0}^{d} h_{j}^{*}\left(\begin{array}{c}n+d-j \\ d\end{array}\right)$ for complex values $h_{j}^{*}$, see [5]. The vector $\left(h_{0}^{*}, h_{1}^{*}, \ldots, h_{d}^{*}\right)$ is called the $h^{*}$-vector of $P$, and a celebrated theorem of Stanley confirms that $h_{j}^{*}$ are nonnegative integers for all $j$, [12, Theorem 2.1].

In addition to positivity, we have verified that the coefficients $\left(\Delta^{j} p_{S}\right)(m)$ are log-concave in $j$ for all admissible sets $S$ with $m=\max (S) \leq 20$, and we suspect that log-concavity holds in general. We note that log-concavity along with our positivity result would imply the unimodality of the coefficients $\left(\Delta^{j} p_{S}\right)(m)$ for $1 \leq j \leq m-1$. If unimodality is not true in general, a related problem would be classifying peak sets for which unimodality holds. Such problems are a major theme throughout combinatorics (for instance, they are central in Ehrhart theory [5]) and could lead to many interesting and fruitful combinatorial questions.

In addition, Theorem 1.2 provides supporting evidence for Billey, Fahrbach, and Talmage's stronger conjecture bounding the moduli of the zeros of peak polynomials [4, Conjecture 1.6]. After stating that conjecture, they noted that Ehrhart, chromatic, and Hilbert polynomials are all examples of polynomials with integer coefficients (in some basis) whose roots are bounded in the complex plane [1, 5, 6, 7, 10, 11. Their conjecture suggests that peak polynomials fit into the family of polynomials sharing these properties.

\section{Peak polynomial positivity}

We begin with a definition that is used throughout the rest of this paper.

Definition 2.1. Let $S=\left\{i_{1}, i_{2}, \ldots, i_{s}\right\} \subseteq[n]$ with $i_{1}<i_{2}<\ldots<i_{s}$ be an $n$-admissible set, and hence $P(S ; n) \neq \emptyset$. For $1 \leq \ell \leq s$ define

$$
\begin{aligned}
S_{i_{\ell}} & =\left\{i_{1}, i_{2}, \ldots, i_{\ell-1}, i_{\ell}-1, i_{\ell+1}-1, i_{\ell+2}-1, \ldots, i_{s}-1\right\}, \\
\widehat{S}_{i_{\ell}} & =\left\{i_{1}, i_{2}, \ldots, i_{\ell-1}, \widehat{i_{\ell}}, i_{\ell+1}-1, i_{\ell+2}-1, \ldots, i_{s}-1\right\}
\end{aligned}
$$

where the notation $\widehat{i_{\ell}}$ means that the element $i_{\ell}$ has been omitted from the set. 
In general, the sets $S_{i_{\ell}}$ might not be $n$-admissible as they may contain two adjacent integers when $i_{\ell}-1=i_{\ell-1}+1$. However, the sets $\widehat{S}_{i_{\ell}}$ are always $n$-admissible.

Example 2.2. If $S=\{3,5,8\} \subseteq[9]$, then

$$
\begin{array}{lll}
S_{3}=\{2,4,7\}, & S_{5}=\{3,4,7\}, & S_{8}=\{3,5,7\}, \\
\widehat{S}_{3}=\{4,7\}, & \widehat{S}_{5}=\{3,7\}, & \widehat{S}_{8}=\{3,5\} .
\end{array}
$$

The sets $S_{3}, S_{8}, \widehat{S}_{3}, \widehat{S}_{5}, \widehat{S}_{8}$ are 9 -admissible whereas $S_{5}$ is not.

Our first result describes a recursive construction of the set $P(S ; n+1)$ from disjoint subsets in $\mathfrak{S}_{n}$.

Theorem 2.3. Let $S=\left\{i_{1}, i_{2}, \ldots, i_{s}\right\} \subseteq \mathbb{N}$ with $i_{1}<i_{2}<\ldots<i_{s}$ be a nonempty -admissible set for any $n \geq \max (S)$. Then

$$
|P(S ; n+1)|=2|P(S ; n)|+2 \sum_{\ell=1}^{s}\left|P\left(S_{i_{\ell}} ; n\right)\right|+\sum_{\ell=1}^{s}\left|P\left(\widehat{S}_{i_{\ell}} ; n\right)\right| .
$$

for $n \geq \max (S)$.

Proof. We recursively build all permutations in $P(S ; n+1) \subseteq \mathfrak{S}_{n+1}$ from permutations in $\mathfrak{S}_{n}$ by inserting the number $n+1$ (in different positions) in the permutations of $\mathfrak{S}_{n}$. Let $\pi=\pi_{1} \cdots \pi_{n}$ be a permutation in $\mathfrak{S}_{n}$ and consider the following five cases:

Case 1: If $\pi \in P(S ; n)$, then by inserting $n+1$ after $\pi_{n}$ we create the permutation

$$
\hat{\pi}=\pi_{1} \pi_{2} \cdots \pi_{n}(n+1) \in P(S ; n+1) .
$$

Case 2: If $\pi \in P(S ; n)$, then by inserting $n+1$ between $\pi_{i_{s}-1}$ and $\pi_{i_{s}}$ we create the permutation

$$
\hat{\pi}=\pi_{1} \cdots \pi_{i_{s}-1}(n+1) \pi_{i_{s}} \cdots \pi_{n} \in P(S ; n+1) .
$$

Case 3: If $\pi \in P\left(S_{i_{\ell}} ; n\right)$ for any $1 \leq \ell \leq s$, then by inserting $n+1$ between $\pi_{i_{\ell}-1}$ and $\pi_{i_{\ell}}$ we create the permutation

$$
\hat{\pi}=\pi_{1} \cdots \pi_{i_{\ell}-1}(n+1) \pi_{i_{\ell}} \cdots \pi_{n} \in P(S ; n+1) .
$$

Case 4.1: If $\pi \in P\left(S_{i_{\ell}} ; n\right)$ and $1<\ell \leq s$, then $\pi$ has a peak at position $i_{\ell-1}$ and by inserting $n+1$ between $\pi_{i_{\ell-1}-1}$ and $\pi_{i_{\ell-1}}$ we create the permutation

$$
\hat{\pi}=\pi_{1} \cdots \pi_{i_{\ell-1}-1}(n+1) \pi_{i_{\ell-1}} \cdots \pi_{n} \in P(S ; n+1) .
$$

Case 4.2: If $\pi \in P\left(S_{i_{1}} ; n\right)$ where $S_{i_{1}}=\left\{i_{1}-1, i_{2}-1, \ldots, i_{s}-1\right\}$, then by inserting $n+1$ to the left of $\pi_{1}$ we create the permutation

$$
\hat{\pi}=(n+1) \pi_{1} \cdots \pi_{n} \in P(S ; n+1) .
$$

Case 5: If $\pi \in P\left(\widehat{S}_{i_{\ell}} ; n\right)$ for any $1 \leq \ell \leq s$, then $\pi$ has no peak at position $i_{\ell}$. By inserting $n+1$ between $\pi_{i_{\ell}-1}$ and $\pi_{i_{\ell}}$ we create the permutation

$$
\hat{\pi}=\pi_{1} \cdots \pi_{i_{\ell}-1}(n+1) \pi_{i_{\ell}} \cdots \pi_{n} \in P(S ; n+1) .
$$


The permutations $\hat{\pi}$ created via Cases 1 through 5 are distinct elements of $P(S ; n+1)$. To see this note that if two permutations are the same, when you remove $n+1$ from each they will stay the same, hence they will have the same peak set. Thus the only potential collisions are between Cases 1 and 2, or between Cases 3 and 4. In both cases the permutations in question are distinct because $n+1$ appears in different positions.

In fact, we show that $P(S ; n+1)$ is precisely the union of the permutations $\hat{\pi}$ appearing in Cases 1 through 5 . If this is the case, the sets being disjoint gives us

$$
|P(S ; n+1)|=2|P(S ; n)|+2 \sum_{\ell=1}^{s}\left|P\left(S_{i_{\ell}} ; n\right)\right|+\sum_{\ell=1}^{s}\left|P\left(\widehat{S}_{i_{\ell}} ; n\right)\right| .
$$

Note that any permutation $\hat{\pi}$ in $P(S ; n+1)$ has the number $n+1$ in one of the following positions: $1, i_{1}, \ldots, i_{s}, n+1$. If $n+1$ is in position $n+1$, then removing it from the permutation $\hat{\pi}$ yields a permutation $\pi$ in Case 1. If $n+1$ is in the first position, then removing it from the permutation $\hat{\pi}$ yields a permutation $\pi$ in Case 4.2. If $n+1$ is in position $i_{\ell}$ for some $1 \leq \ell \leq s$, then removing it from the permutation $\hat{\pi}$ leads to three possibilities: a permutation with a peak at position $i_{\ell}$ (Cases 2 and 4.1), a permutation with a peak at position $i_{\ell}-1$ (Case 3 ), or a permutation without a peak at positions $i_{\ell}-1$ or $i_{\ell}$ (Case 5$)$. Thus we have created all permutation in $P(S ; n+1)$ via the constructions in Cases 1-5.

Note that the recurrence provided in Theorem 2.3 also holds whenever $S=\emptyset$ as the only contributing term is $2|P(\emptyset ; n)|$. The following result plays a key role in the proof of Theorem 1.2 .

Corollary 2.4. Let $S=\left\{i_{1}, i_{2}, \ldots, i_{s}\right\} \subseteq[n]$ with $i_{1}<i_{2}<\ldots<i_{s}$ be a nonempty $n$-admissible set. Then the following equality of polynomials holds

$$
\left(\Delta p_{S}\right)(x)=\sum_{\ell=1}^{s} p_{S_{i_{\ell}}}(x)+\sum_{\ell=1}^{s} p_{\widehat{S}_{i_{\ell}}}(x) .
$$

Proof. Let $m=\max (S)$. It suffices to show that the two polynomials agree at infinitely many values, and to do so we show that for any $q \geq m$,

$$
\left(\Delta p_{S}\right)(q)=\sum_{\ell=1}^{s} p_{S_{i_{\ell}}}(q)+\sum_{\ell=1}^{s} p_{\widehat{S}_{i_{\ell}}}(q) .
$$

Observe that for such $q$, substituting Equation (1) appropriately into Theorem 2.3 yields

$$
\begin{aligned}
2^{q-|S|} p_{S}(q+1)-2^{q-|S|} p_{S}(q) & =\sum_{\ell=1}^{s} 2^{q-\left|S_{i_{\ell}}\right|} p_{S_{i_{\ell}}}(q)+\sum_{\ell=1}^{s} 2^{q-\left|\widehat{S}_{i_{\ell}}\right|-1} p_{\widehat{S}_{i_{\ell}}}(q) \\
& =2^{q-|S|} \sum_{\ell=1}^{s} p_{S_{i_{\ell}}}(q)+2^{q-|S|} \sum_{\ell=1}^{s} p_{\widehat{S}_{i_{\ell}}}(q)
\end{aligned}
$$

where the last equality holds since $\left|S_{i_{\ell}}\right|=|S|$ and $\left|\widehat{S}_{i_{\ell}}\right|=|S|-1$ for all $1 \leq \ell \leq s$. The result follows from multiplying Equation (6) by $1 / 2^{q-|S|}$.

The following lemma reduces proving Theorem 1.2 to the case when $k=\max (S)$. 
Lemma 2.5. Let $S=\left\{i_{1}, i_{2}, \ldots, i_{s}\right\} \subseteq[n]$ with $i_{1}<i_{2}<\ldots<i_{s}$ be a nonempty $n$-admissible set, and $m=i_{s}$. If $\left(\Delta^{j} p_{S}\right)(m)>0$ for all $1 \leq j \leq m-1$, then $\left(\Delta^{j} p_{S}\right)(k)>0$ for all $k \geq m$ and all $1 \leq j \leq m-1$.

Proof. Using the definition of the operator $\Delta$ we obtain $\left(\Delta^{j} p_{S}\right)(k+1)=\left(\Delta^{j} p_{S}\right)(k)+\left(\Delta^{j+1} p_{S}\right)(k)$. For $1 \leq j \leq m-2$ the proof follows by induction on $k$ using $k=m$ as the base case. For $j=m-1$, we get $\left(\Delta^{m-1} p_{S}\right)(k+1)=\left(\Delta^{m-1} p_{S}\right)(k)+\left(\Delta^{m} p_{S}\right)(k)$. Since $\operatorname{deg}\left(p_{S}\right)=m-1$ and the operator $\Delta$ decreases the degree of a polynomial by one, then $\left(\Delta^{m} p_{S}\right)(x)=0$ and the equation becomes $\left(\Delta^{m-1} p_{S}\right)(k+1)=\left(\Delta^{m-1} p_{S}\right)(k)$. Thus, recursively we get $\left(\Delta^{m-1} p_{S}\right)(k)=\left(\Delta^{m-1} p_{S}\right)(m)>0$ for all $k \geq m$.

We are now ready to prove the positivity conjecture for peak polynomials.

Proof of Theorem 1.2. Using Lemma 2.5 it is enough to prove the theorem when $k=\max (S)$. Billey, Burdzy, and Sagan showed that the result is true when $|S|=1$ [3, Proposition 16].

Suppose now that $|S| \geq 2$, and let $m=\max (S)$. From Corollary 2.4 we have

$$
\left(\Delta p_{S}\right)(x)=\sum_{\ell=1}^{s} p_{S_{i_{\ell}}}(x)+\sum_{\ell=1}^{s} p_{\widehat{S}_{i_{\ell}}}(x) .
$$

Note that in the above equation, each term indexed by a non-admissible set $S_{i_{\ell}}$ is zero. However, the sets $\widehat{S}_{i_{\ell}}$ indexing the terms in the second sum are admissible. For all such sets $\widehat{S}_{i_{\ell}}$ we have the following inequality $\max \left(\widehat{S}_{i_{\ell}}\right)<\max (S)=m$. Theorem 3.8 and Proposition 4.4 in [4] imply that

$$
0=p_{\widehat{S}_{i_{\ell}}}\left(\max \left(\widehat{S}_{i_{\ell}}\right)\right)<p_{\widehat{S}_{i_{\ell}}}(m) .
$$

Similarly, for all admissible sets $S_{i_{\ell}}$ we have that $\max \left(S_{i_{\ell}}\right)<\max (S)=m$, and $p_{S_{i_{\ell}}}(m)>0$. Using Equation (7) we conclude that $\left(\Delta p_{S}\right)(m)>0$, which proves the theorem when $j=1$.

Suppose then that $2 \leq j \leq m-1$ and let $S$ be any admissible set. We proceed by induction on $m=\max (S)$. The base case is when $S=\{2\}$. This case, as well as the cases where $|S|=1$, are proved in [3, Proposition 16]. Thus, for the rest of the proof, we assume $|S| \geq 2$.

Suppose the result is true for all nonempty admissible sets $T$ with $\max (T)<\max (S)$. By applying the $\Delta$ operator repeatedly to Equation (7) we get that,

$$
\left(\Delta^{j} p_{S}\right)(m)=\left(\Delta\left(\Delta^{j-1} p_{S}\right)\right)(m)=\sum_{\ell=1}^{s}\left(\Delta^{j-1} p_{S_{i_{\ell}}}\right)(m)+\sum_{\ell=1}^{s}\left(\Delta^{j-1} p_{\widehat{S}_{i_{\ell}}}\right)(m) .
$$

Since $\operatorname{deg}\left(p_{T}(x)\right)=\max (T)-1$ for any nonempty admissible set $T \subseteq[n]$ and $\operatorname{deg}\left(p_{T}(x)\right)=0$ when $T$ is empty or non-admissible, in particular, we have that for all $1 \leq \ell \leq s$

$$
\operatorname{deg}\left(p_{S}(x)\right)>\operatorname{deg}\left(p_{\widehat{S}_{i_{\ell}}}(x)\right) \quad \text { and } \quad \operatorname{deg}\left(p_{S}(x)\right)>\operatorname{deg}\left(p_{S_{i_{\ell}}}(x)\right) .
$$

Applying the inductive hypothesis to the sets $\widehat{S}_{i_{\ell}}$ and $S_{i_{\ell}}$ we conclude that,

$$
\Delta^{j-1}\left(p_{S_{i_{\ell}}}\right)(m) \geq 0 \quad \text { and } \Delta^{j-1}\left(p_{\widehat{S}_{i_{\ell}}}\right)(m) \geq 0,
$$

where we get equality only when the sets indexing the polynomials are non-admissible, or when the degree of the polynomial is less than $j-1$ in which case applying $\Delta$ a total of $j-1$ times leads to 
the zero polynomial. To finish the proof, using Equation (8) and the above inequalities, it is enough to show that there is at least one set for which the inequality is strict. Write $S=\left\{i_{1}, i_{2}, \ldots, i_{s}\right\}$ with $i_{1}<i_{2}<\cdots<i_{s}$. If $i_{s}-i_{s-1}>2$ then the set $S_{i_{s}}=\left\{i_{1}, i_{2}, \ldots, i_{s-1}, i_{s}-1\right\}$ is admissible and

$$
\operatorname{deg}\left(p_{S_{i_{s}}}\right)=\operatorname{deg}(S)-1=m-2 \geq j-1, \text { thus } \Delta^{j-1}\left(p_{S_{i_{s}}}\right)(m)>0 .
$$

If $i_{s}-i_{s-1}=2$ then $\widehat{S}_{i_{s-1}}=\left\{i_{1}, i_{2}, \ldots, i_{s-2}, i_{s}-1\right\}$ is admissible and

$$
\operatorname{deg}\left(p_{\widehat{S}_{i_{s-1}}}\right)=\operatorname{deg}(S)-1=m-2 \geq j-1, \text { thus } \Delta^{j-1}\left(p_{\widehat{S}_{i_{s-1}}}\right)(m)>0 .
$$

Finally, as mentioned in the proof of Lemma 2.5, the operator $\Delta$ decreases the degree of a polynomial by one, thus $\left(\Delta^{m} p_{S}\right)(x)=0$.

\section{Acknowledgments}

We thank Sara Billey for introducing us to this problem, for many helpful conversations, and for insightful comments regarding an earlier version of this manuscript. We thank the anonymous referees for their thorough consideration and helpful comments. We thank Lucas Everham, Vince Marcantonio, Karen Marino, and Darleen Perez-Lavin for beneficial conversations regarding this topic. This work was supported through mini-collaboration travel grants via awards from the National Science Foundation (DMS-1545136) and the National Security Agency (H98230-15-1-0091). The second author gratefully acknowledges travel support from the United States Military Academy. The third author gratefully acknowledges funding support from the Seidler Student/Faculty Undergraduate Scholarly Collaboration Fellowship Program at Florida Gulf Coast University.

\section{References}

[1] M. Beck, J. A. De Loera, M. Develin, J. Pfeifle, and R. P. Stanley. Coefficients and roots of ehrhart polynomials. Integer Points in Polyhedra-Geometry, Number Theory, Representation Theory, Algebra, Optimization, Statistics, Contemporary Mathematics, 374:15-36, 2005.

[2] S. Billey, K. Burdzy, S. Pal, and B. Sagan. On meteors, earthworms and WIMPs. Ann. Appl. Probab., 25(4):1729-1779, 2015.

[3] S. Billey, K. Burdzy, and B. Sagan. Permutations with given peak set. J. of Integer Seq., 16, 2013.

[4] S. Billey, M. Fahrbach, and A. Talmage. Coefficients and Roots of Peak Polynomials. Exp. Math., 25(2):165-175, 2016.

[5] B. Braun and M. Develin. Ehrhart polynomial roots and stanley's non-negativity theorem. Integer Points in Polyhedra-Geometry, Number Theory, Representation Theory, Algebra, Optimization, Statistics, Contemporary Mathematics, 452:67-78, 2008.

[6] F. Brenti. Expansions of chromatic polynomials and log-concavity. Transactions of the American Mathematical Society, 332(2):729-756, 1992. 
[7] F. Brenti, G. F. Royle, and D. G. Wagner. Location of zeros of chromatic and related polynomials in graphs. Canadian Journal of Mathematics, 46(1):55-80, 1994.

[8] F. Castro-Velez, A. Diaz-Lopez, R. Orellana, J. Pastrana, and R. Zevallos. Number of permutations with same peak set for signed permutations. arXiv:1308.6621, 2014.

[9] A. Diaz-Lopez, P. E. Harris, E. Insko, and D. Perez-Lavin. Peak Sets of Classical Coxeter Groups. Involve, to appear.

[10] J. Pfeifle. Gale duality bounds for roots of polynomials with nonnegative coefficients. Journal of Combinatorial Theory Series A, (117):248-271, 2010.

[11] F. Rodriguez-Villegas. On the zeros of certain polynomials. Proceedings of the American Mathematical Society, 130(8):2251-2254, 2002.

[12] R. P. Stanley. Decompositions of rational convex polytopes. Annals of Discrete Mathematics, 6:333-342, 1980. 